\title{
First Monte Carlo Global Analysis of Nucleon Transversity with Lattice QCD Constraints
}

\author{
H.-W. Lin, ${ }^{1}$ W. Melnitchouk, ${ }^{2}$ A. Prokudin, ${ }^{2,3}$ N. Sato, ${ }^{4}$ and H. Shows III ${ }^{5}$ \\ (Jefferson Lab Angular Momentum (JAM) Collaboration) \\ ${ }^{1}$ Michigan State University, East Lansing, Michigan 48824, USA \\ ${ }^{2}$ Jefferson Lab, Newport News, Virginia 23606, USA \\ ${ }^{3}$ Penn State Berks, Reading, Pennsylvania 19610, USA \\ ${ }^{4}$ University of Connecticut, Storrs, Connecticut 06269, USA \\ ${ }^{5}$ Louisiana State University, Baton Rouge, Louisiana 70803, USA
}

(Received 27 October 2017; published 11 April 2018)

\begin{abstract}
We report on the first global QCD analysis of the quark transversity distributions in the nucleon from semi-inclusive deep-inelastic scattering (SIDIS), using a new Monte Carlo method based on nested sampling and constraints on the isovector tensor charge $g_{T}$ from lattice QCD. A simultaneous fit to the available SIDIS Collins asymmetry data is compatible with $g_{T}$ values extracted from a comprehensive reanalysis of existing lattice simulations, in contrast to previous analyses, which found significantly smaller $g_{T}$ values. The contributions to the nucleon tensor charge from $u$ and $d$ quarks are found to be $\delta u=0.3(2)$ and $\delta d=-0.7(2)$ at a scale $Q^{2}=2 \mathrm{GeV}^{2}$.

DOI: 10.1103/PhysRevLett.120.152502
\end{abstract}

Along with the unpolarized $\left(f_{1}\right)$ and helicity-dependent $\left(g_{1}\right)$ parton distribution functions (PDFs), the transversity distribution $\left(h_{1}\right)$ completes the full set of quark PDFs that characterize the collinear structure of the nucleon at leading twist. While considerable information has been accumulated on the first two distributions from several decades of deep-inelastic scattering (DIS) and other high-energy experiments [1-4], comparatively little is known about the transversity PDFs. The transversity PDF, $h_{1}^{q}(x)$, gives the distribution of a transversely polarized quark $q$ carrying a momentum fraction $x$ in a transversely polarized nucleon, and its lowest moment, $\delta q \equiv \int_{0}^{1} d x\left[h_{1}^{q}(x)-h_{1}^{\bar{q}}(x)\right]$, gives the nucleon's tensor charge for quark $q$ [5-11]. In addition to providing fundamental information on the quark spin structure of the nucleon, the tensor charge also plays an important role in constraining hadronic physics backgrounds in probes of physics beyond the standard model [12-14].

Compared with the chiral-even $f_{1}$ and $g_{1}$ PDFs, the experimental exploration of the chiral-odd $h_{1}$ is considerably more involved, requiring the coupling of the transversity distribution to another chiral-odd function [6]. Observables sensitive to transversity include the Collins

Published by the American Physical Society under the terms of the Creative Commons Attribution 4.0 International license. Further distribution of this work must maintain attribution to the author(s) and the published article's title, journal citation, and DOI. Funded by SCOAP ${ }^{3}$. single-spin asymmetries in semi-inclusive deep-inelastic scattering (SIDIS), where $h_{1}$ couples to the chiral-odd Collins fragmentation function (FF) $H_{1}^{\perp}$ [15], while two Collins FFs generate an azimuthal asymmetry in twohadron production in $e^{+} e^{-}$annihilation [16].

Several previous analyses have attempted to extract the transverse momentum dependent (TMD) transversity distributions from both SIDIS and $e^{+} e^{-}$data. Anselmino et al. [17-19] employed a factorized Gaussian ansatz to relate the TMD distributions to the $h_{1}^{q}$ PDFs, while Kang et al. [20,21] used, in addition, the TMD evolution formalism [22]. In both cases, the $x$ dependence of $h_{1}^{q}(x)$ was parametrized in terms of the sum of unpolarized and helicity distributions at the initial scale. Working within collinear factorization, Bacchetta et al. [23-25] also extracted transversity PDFs from pion pair production in SIDIS using dihadron FFs from $e^{+} e^{-}$data. These analyses gave values for the isovector moment $g_{T} \equiv \delta u-\delta d$ in the range 0.4-1, with sizeable $(30 \%-50 \%)$ uncertainties. In all of these studies, the experimental coverage was restricted to the region $0.02 \lesssim x \lesssim 0.3$, so that the determination of the full moment required extrapolation outside the measured range.

Complementing the challenging empirical extractions of transversity, first-principles lattice QCD calculations can provide additional information on the nucleon transverse spin structure. While recent breakthroughs in quasi-PDFs have allowed the first direct lattice computations of the $x$ dependence of transversity [26,27], calculations of moments of the isovector $h_{1}^{q} \mathrm{PDF}$ are more developed, 
with a number of simulations of $g_{T}$ having been performed [28-34] at physical pion masses and with multiple lattice spacings and volumes. No significant contamination from excited states has been observed, along with very mild volume and lattice spacing dependence, making $g_{T}$ a "golden" channel in lattice nucleon structure studies. Curiously, however, all of the simulations give values of $g_{T}$ close to unity, in contrast to the phenomenological values, which are generally smaller [10,21], with central values $\sim 0.5-0.6$. This prompts the question of whether the systematic differences between the lattice and phenomenological results suggest a real tension between the two. From the uncertainties found by Kang et al. [21], for example, one would conclude that after the inclusion of data from the future SoLID experiment at Jefferson Lab [10], the phenomenological values of $g_{T}$ would be incompatible with lattice at more than $5 \sigma$ C.L.

In this Letter, we address the question of whether the experimental data on transversity are compatible with the lattice $g_{T}$ results-whether there indeed is a "transverse-spin puzzle," as suggested by some of the previous analyses $[10,21]$ - by using the lattice data on $g_{T}$ as an additional constraint on the global QCD analysis of transversity. We implement several important improvements over previous analyses, making use of a more robust fitting methodology based on Monte Carlo (MC) sampling methods. Specifically, we use the nested sampling algorithm [35-37], which maps the likelihood function into an $\mathrm{MC}$-weighted parameter sample and allows a rigorous determination of PDF uncertainties. This approach improves the fitting methodology of Refs. [20,21] by allowing more flexible parametrizations of the initial conditions of the transversity and Collins FFs. Similar MC-based methods have recently been used to analyze collinear PDFs [38,39] and FFs [39,40], but they have never before been applied to TMDs.

To begin with, we revisit the existing lattice QCD simulations of $g_{T}$ to obtain a reliable averaged data point that can be used in the global QCD analysis. One challenge is that the various lattice calculations estimate systematic uncertainties differently, making it problematic to simply average the reported values. We instead combine the available dynamical simulation data, using only calculations with multiple lattice spacings, volumes and quark masses; we use several procedures to ensure that the final uncertainties are not underestimated.

There are three available data sets that meet these criteria: the PNDME Collaboration results with $N_{f}=2+$ $1+1$ flavors [28], the RQCD Collaboration data with $N_{f}=2$ [33], and the LHPC set with $N_{f}=2+1$ [30]. Cuts on the data are imposed for pion masses $m_{\pi}^{2}<0.12 \mathrm{GeV}^{2}$ and for $m_{\pi} L>3$, where $L^{3}$ is the lattice volume, to control the chiral and infinite-volume extrapolations. Since all of the lattice simulations show a mild dependence on the volume and lattice spacing $a$, the simplest approach is to extrapolate $g_{T}$ considering only the $m_{\pi}$ dependence. Extrapolating the data either linearly in $m_{\pi}^{2}$ or including chiral logarithms $\left(\sim m_{\pi}^{2} \ln m_{\pi}^{2}\right)$, as predicted from chiral effective theory [41,42], gives $g_{T}^{\text {latt }}=1.006(22)$.

To further include uncertainties from taking the continuum limit, we assign a different lattice discretization extrapolation coefficient for each simulation [28,30,33]. To account for the different actions, we use $\mathcal{O}(a)$ for the PNDME and LHPC results, and $\mathcal{O}\left(a^{2}\right)$ for RQCD. For the volume dependence, we consider both $e^{m_{\pi} L}$ and $m_{\pi}^{2} e^{m_{\pi} L}$ forms. Taking all possible combinations then gives 12 distinct fitting formulas for the continuum extrapolation of $g_{T}$. The results of these fits are combined using the Akaike information criterion, $\mathrm{AIC}=2 k+\chi^{2}$, where $k$ is the number of free parameters in the fit and $\chi^{2}$ is the minimum sum of squared fit residuals. Each fit is weighted by the factor $w_{i}=P_{i} /\left(\sum_{j} P_{j}\right)$, where $P_{j}=\exp \left[-\left(\mathrm{AIC}_{j}-\min \mathrm{AIC}\right) / 2\right]$, which yields $g_{T}^{\text {latt }}=1.008(56)$.

Another approach is to average the lattice data using methods advocated by the Flavor Lattice Averaging Group (FLAG) [43]. However, given that most extrapolations of nucleon matrix elements do not explicitly control finite volume and lattice spacing systematics, such an averaging will be dominated by results with the most optimistic systematic uncertainty estimates. We extrapolate each group's data using a single, universal formula, assuming linear dependence on $m_{\pi}^{2}, e^{m_{\pi} L}$ and $a$ (or $a^{2}$ ), and then perform a weighted analysis as in the FLAG approach. The result is $g_{T}^{\text {latt }}=1.00(5)$, which is consistent with the above estimate. To be conservative, we take the larger uncertainty, $g_{T}^{\text {latt }}=1.01(6)$, as the final averaged value to be used in the global analysis.

For the experimental data used in our fit, we consider the $\sin \left(\phi_{h}+\phi_{s}\right)$ modulation of the differential SIDIS cross section, or Collins asymmetry,

$$
A_{U T}^{\sin \left(\phi_{\mathrm{h}}+\phi_{\mathrm{s}}\right)}=\frac{2(1-y)}{1+(1-y)^{2}} \frac{F_{U T}^{\sin \left(\phi_{h}+\phi_{s}\right)}}{F_{U U}},
$$

where $\phi_{h}$ and $\phi_{s}$ are the azimuthal angles for the transverse momentum of the produced hadron $h$ and the nucleon spin vector with respect to the lepton plane in the virtual photon-nucleon center of mass frame, and $y$ is the fractional energy loss of the incident lepton. The structure functions $F_{U U}$ and $F_{U T}^{\sin \left(\phi_{h}+\phi_{s}\right)}$ are functions of the Bjorken variable $x=Q^{2} / 2 P \cdot q$, the hadron momentum fraction $z=P \cdot P_{h} / P \cdot q$, and the hadron transverse momentum $P_{h \perp}$, where $P, P_{h}$ and $q$ are the four-momenta of the target, produced hadron, and exchanged photon, respectively, and $Q^{2}=-q^{2}$. For $P_{h \perp} \ll Q$ these can be written as convolutions of the unpolarized $f_{1}^{q}$ TMD PDF and unpolarized $D_{1}^{h / q}$ TMD FF, and the TMD transversity PDF $h_{1}^{q}$ and $H_{1}^{\perp h / q}$ (Collins) FF, respectively, 


$$
\begin{gathered}
F_{U U}=\mathcal{C}\left(f_{1} \otimes D_{1}\right), \\
F_{U T}^{\sin \left(\phi_{h}+\phi_{s}\right)}=\mathcal{C}\left(\frac{\hat{\boldsymbol{h}} \cdot \boldsymbol{p}_{\perp}}{z m_{h}} \otimes h_{1} \otimes H_{1}^{\perp}\right),
\end{gathered}
$$

where $\mathcal{C}$ is the standard TMD convolution operator [44], $\hat{\boldsymbol{h}}$ is a unit vector along $\boldsymbol{P}_{h \perp}$, and $\boldsymbol{p}_{\perp}$ is the transverse momentum of $h$ with respect to the fragmenting quark.

The TMD PDFs depend on $x$ and the parton transverse momentum $k_{\perp}$, while the FFs depend on $z$ and $p_{\perp}$, with their $Q^{2}$ dependence governed by the Collins-Soper equations [22,45]. The existing data on Collins asymmetries have very mild dependence on $Q^{2}$ and are compatible with no evolution $[21,46]$. For the parametrization of the unpolarized and transversity TMD PDFs, we follow Refs. [17-19] in adopting a factorized form,

$$
f^{q}\left(x, k_{\perp}^{2}\right)=f^{q}(x) \mathcal{G}_{f}^{q}\left(k_{\perp}^{2}\right),
$$

where the generic function $f^{q}=f_{1}^{q}$ or $h_{1}^{q}$, and the $k_{\perp}^{2}$ dependence is given by a Gaussian distribution,

$$
\mathcal{G}_{f}^{q}\left(k_{\perp}^{2}\right)=\frac{1}{\pi\left\langle k_{\perp}^{2}\right\rangle_{f}^{q}} \exp \left[-\frac{k_{\perp}^{2}}{\left\langle k_{\perp}^{2}\right\rangle_{f}^{q}}\right] .
$$

The transverse widths $\left\langle k_{\perp}^{2}\right\rangle_{f}^{q}$ are in general flavor dependent, and can be functions of $x$, although here we assume their $x$ dependence is negligible. For the TMD FFs, the unpolarized distribution is parametrized analogously,

$$
D_{1}^{h / q}\left(z, p_{\perp}^{2}\right)=D_{1}^{h / q}(z) \mathcal{G}_{D_{1}}^{h / q}\left(p_{\perp}^{2}\right),
$$

while the Collins FF involves an additional $z$ dependent weight factor,

$$
H_{1}^{\perp h / q}\left(z, p_{\perp}\right)=\frac{2 z^{2} m_{h}^{2}}{\left\langle p_{\perp}^{2}\right\rangle_{H_{1}^{\perp}}^{h / q}} H_{1 h / q}^{\perp(1)}(z) \mathcal{G}_{H_{1}^{\perp}}^{h / q}\left(p_{\perp}^{2}\right) .
$$

The $p_{\perp}^{2}$ dependence of the functions $\mathcal{G}_{D_{1}}^{h / q}$ and $\mathcal{G}_{H_{1}^{\perp}}^{h / q}$ is assumed to be Gaussian, in analogy with (5), with the average $\left\langle p_{\perp}^{2}\right\rangle^{h / q}$ independent of $z$. The $z$ dependence of the Collins FF is parametrized in terms of its $p_{\perp}^{2}$-weighted moment, $H_{1 h / q}^{\perp(1)}(z)$ [21]. Using the TMD PDFs and FFs in Eqs. (4)-(7), the $P_{h \perp}^{2}$ dependence in the structure functions is then proportional to $\exp \left(-P_{h \perp}^{2} /\left\langle P_{h \perp}^{2}\right\rangle_{f, D}^{h / q}\right)$, where $\left\langle P_{h \perp}^{2}\right\rangle_{f, D}^{q}=z^{2}\left\langle k_{\perp}^{2}\right\rangle_{f}^{q}+\left\langle p_{\perp}^{2}\right\rangle_{D}^{h / q}$.

Our global analysis fits SIDIS $\pi^{ \pm}$production data from proton and deuteron targets, including their $x, z$, and $P_{h \perp}$ dependence, with a total of 106 data points from the HERMES [47] and COMPASS [48,49] experiments. This gives four linear combinations of transversity TMD
PDFs and Collins TMD FFs for different quark flavors, from which we extract the $u, d$, and antiquark transversity PDFs (from four $x$-dependent combinations) and the favored and unfavored Collins FFs (from four $z$-dependent combinations), together with their respective transverse momentum widths (from the $P_{h \perp}$ dependence). We do not include lower-energy Collins asymmetry data from Jefferson Lab on ${ }^{3} \mathrm{He}$ nuclei because of concerns about the separation of the current and target fragmentation regions at relatively low energies [50].

In selecting the data to be used in the fit, we place several kinematic cuts on the $z, P_{h \perp}$, and $Q^{2}$ dependencies in order to isolate samples where the theoretical framework used in this analysis is applicable. To stay within the current fragmentation region, only data for $z>0.2$ are included, and to avoid contamination from vector-meson production and soft-gluon effects, we exclude data above $z=0.6$. For the $P_{h \perp}$ dependence, we exclude the regions where $P_{h \perp}$ is very small $\left(P_{h \perp}>0.2 \mathrm{GeV}\right)$ or very large $\left(P_{h \perp}<0.9 \mathrm{GeV}\right)$ : the former to avoid acceptance issues for the lowest $P_{h \perp}$ bin of the HERMES multiplicity data, and the latter to ensure the applicability of the Gaussian assumption, without the need for introducing the $Y$ term [50]. To stay above the charm threshold, we restrict ourselves to $Q^{2}>m_{c}^{2}$.

Because the existing SIDIS Collins asymmetry data have a rather small $Q^{2}$ range, and $Q^{2}$ evolution effects tend to cancel in ratios, there is no clear empirical indication of scale dependence in the asymmetries. It is a reasonable approximation, therefore, to neglect the $Q^{2}$ dependence in the $F_{U T}^{\sin \left(\phi_{h}+\phi_{s}\right)}$ structure function, and freeze the scale in the unpolarized $f_{1}^{q}$ and $D_{1}^{q}$ distributions in $F_{U U}$ at a value $Q^{2}=$ $2 \mathrm{GeV}^{2}$ that is typical of SIDIS data. (In contrast, since $e^{+} e^{-}$data are taken at higher energies, neglecting the scale dependence between the $e^{+} e^{-}$and SIDIS measurements would introduce uncontrolled errors from not including the full TMD evolution where its effects may be important).

In determining the transversity TMDs $h_{1}^{q}\left(x, k_{\perp}^{2}\right)$, we parametrize the $x$ dependence by the form $h_{1}^{q}(x)=$ $N_{q} x^{a_{q}}(1-x)^{b_{q}}$ for each of the flavors $q=u, d$, and $\bar{q}$, assuming a symmetric sea, $h_{1}^{\bar{u}}=h_{1}^{\bar{d}}=h_{1}^{s}=h_{1}^{\bar{s}}$, and use isospin symmetry to relate the distributions in the proton and neutron. For the Collins $\pi^{ \pm}$distributions, we use a similar functional form to parametrize the $z$ dependence of the favored $H_{1 \text { (fav) }}^{\perp(1)} \equiv H_{1 \pi^{+} / u}^{\perp(1)}=H_{1 \pi^{+} / \bar{d}}^{\perp(1)}$ FFs and the unfavored $H_{1 \text { (unf) }}^{\perp(1)}$ FFs for $\{d, \bar{u}, s, \bar{s}\} \rightarrow \pi^{+}$, with the distributions for $\pi^{-}$related by charge conjugation. For the $x$ dependence of the spin-averaged $f_{1}^{q}$ distributions we use the CJ15 leading-order parametrization [51], while for the $z$ dependence of $D_{1}^{q}$, we utilize the leading-order DSS fit [52]. Choosing a different FF parametrization would not affect the results significantly, as changes in the $z$ dependence of the FFs could be compensated by modified widths in the Gaussian $P_{h \perp}$ distributions. 
For the transverse-momentum widths $\left\langle k_{\perp}^{2}\right\rangle_{f}^{q}$ of the TMD PDFs $f_{1}^{q}$ and $h_{1}^{q}$, two Gaussian widths are used, one for the valence type $(q=u, d)$ and one for the sea-quark type $(q=\bar{u}, \bar{d}, s, \bar{s})$ functions. Similarly, for the TMD FFs two Gaussian widths for $\left\langle p_{\perp}^{2}\right\rangle_{D}^{h / q}$ are used, for the favored (such as $u$ or $\bar{d}$ to $\pi^{+}$) and unfavored ( $\bar{u}$ or $d$ to $\pi^{+}$) type of FF. In total, we therefore have 23 parameters to be extracted from data, 19 of which describe $F_{U T}^{\sin \left(\phi_{h}+\phi_{s}\right)}$ and 4 for the transverse part of $F_{U U}$. To determine the latter, we perform an independent fit to the HERMES $\pi^{ \pm}$and $K^{ \pm}$multiplicity data [53], which include 978 data points that survive the same cuts as employed for $A_{U T}^{\sin \left(\phi_{h}+\phi_{s}\right)}$.

Using the nested sampling MC algorithm [35-37], we compute the expectation value $\mathrm{E}[\mathcal{O}]$ and variance $\mathrm{V}[\mathcal{O}]$,

$$
\begin{aligned}
\mathrm{E}[\mathcal{O}] & =\int d^{n} a \mathcal{P}(\boldsymbol{a} \mid \text { data }) \mathcal{O}(\boldsymbol{a}) \simeq \sum_{k} w_{k} \mathcal{O}\left(\boldsymbol{a}_{k}\right), \\
\mathrm{V}[\mathcal{O}] & =\int d^{n} a \mathcal{P}(\boldsymbol{a} \mid \text { data })(\mathcal{O}(\boldsymbol{a})-\mathrm{E}[\mathcal{O}])^{2} \\
& \simeq \sum_{k} w_{k}\left(\mathcal{O}\left(\boldsymbol{a}_{k}\right)-\mathrm{E}[\mathcal{O}]\right)^{2},
\end{aligned}
$$

for each observable $\mathcal{O}$ (such as a TMD or a function of TMDs), which is a function of the $n$-dimensional vector parameters $\boldsymbol{a}$ with probability density $\mathcal{P}(\boldsymbol{a} \mid$ data $)$ [40]. Using Bayes' theorem, the latter is given by

$$
\mathcal{P}(\boldsymbol{a} \mid \text { data })=\frac{1}{Z} \mathcal{L}(\text { data } \mid \boldsymbol{a}) \pi(\boldsymbol{a}),
$$

where $\pi(\boldsymbol{a})$ is the prior distribution for the vector parameters $\boldsymbol{a}$, and

$$
\mathcal{L}(\text { data } \mid \boldsymbol{a})=\exp \left[-\frac{1}{2} \chi^{2}(\boldsymbol{a})\right]
$$

is the likelihood function, with $Z=\int d^{n} a \mathcal{L}($ data $\mid \boldsymbol{a}) \pi(\boldsymbol{a})$ the Bayesian evidence parameter. Using a flat prior, the nested sampling algorithm constructs a set of MC samples $\left\{\boldsymbol{a}_{k}\right\}$ with weights $\left\{w_{k}\right\}$, which are then used to evaluate the integrals in Eqs. (8).

The results of the fit indicate good overall agreement with the Collins $\pi^{+}$and $\pi^{-}$asymmetries, as illustrated in Fig. 1, for both HERMES [47] and COMPASS $[48,49]$ data, with marginally better fits for the latter. The $\chi^{2} /$ datum values for the $\pi^{+}$and $\pi^{-}$data are 28.6/53 and 40.4/53, respectively, for a total of $68.9 / 106 \approx 0.65$. The larger $\chi^{2}$ for $\pi^{-}$stems from the few outlier points in the $x$ and $z$ spectra, as evident in Fig. 1. The SIDIS-only fit is almost indistinguishable, with $\chi_{\text {SIDIS }}^{2}=69.2$. Clearly, our MC results do not indicate any tension between the SIDIS data and lattice QCD calculations of $g_{T}$, nor any "transverse spin problem."
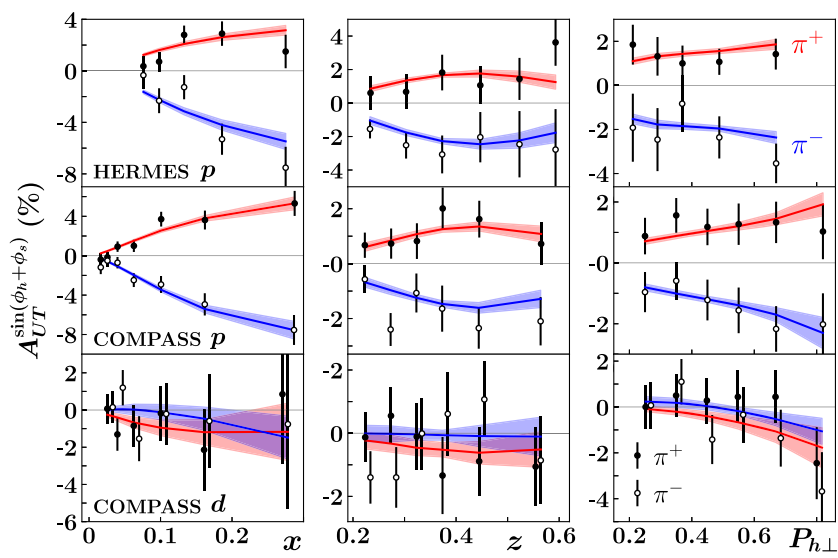

FIG. 1. A comparison of the full SIDIS + lattice fit with the $\pi^{+}$ (filled circles) and $\pi^{-}$(open circles) Collins asymmetries $A_{U T}^{\sin \left(\phi_{h}+\phi_{s}\right)}$ from HERMES [47] and COMPASS [48,49] data (in percent), as a function of $x, z$, and $P_{h \perp}$ (in GeV).

The resulting transversity PDFs $h_{1}^{u}$ and $h_{1}^{d}$ and Collins favored and unfavored FFs, $H_{1 \text { (fav) }}^{\perp(1)}$ and $H_{1 \text { (unf) }}^{\perp(1)}$, are plotted in Fig. 2 for both the SIDIS-only and SIDIS + lattice fits. The positive (negative) sign for the $u(d)$ transversity PDF is consistent with previous extractions, and correlates with the same sign for the Collins FFs in the region of $z$ directly constrained by data. The larger $\left|h_{1}^{d}\right|$ compared with $\left|h_{1}^{u}\right|$ reflects the larger magnitude of the (negative) $\pi^{-}$asymmetry than the (positive) $\pi^{-}$asymmetry. At lower $z$ values, outside the measured region, the uncertainties on the Collins FFs become extremely large. Interestingly, inclusion of the lattice $g_{T}$ datum has very little effect on the central values of the distributions, but reduces significantly the uncertainty bands. The fitted antiquark transversity is consistent with zero, within relatively large uncertainties, and is not shown in Fig. 2.

For the transverse momentum widths, our analysis of the HERMES multiplicities [53] gives a total $\chi^{2} /$ datum of $1079 / 978$, with $\left\langle k_{\perp}^{2}\right\rangle_{f_{1}}^{q}=0.59(1) \mathrm{GeV}^{2}$ and $0.64(6) \mathrm{GeV}^{2}$ for the unpolarized valence and sea quark PDF widths, and $\left\langle p_{\perp}^{2}\right\rangle_{D_{1}}^{\pi / q}=0.116(2) \mathrm{GeV}^{2}$ and $0.140(2) \mathrm{GeV}^{2}$ for the
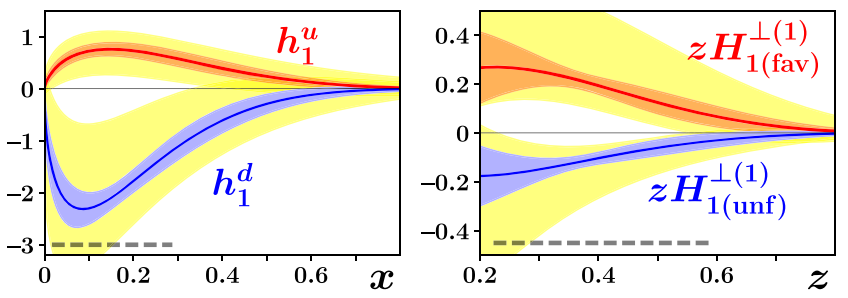

FIG. 2. Transversity PDFs $h_{1}^{u, d}$ and favored $z H_{1 \text { (fav) }}^{\perp(1)}$ and unfavored $z H_{1 \text { (unf) }}^{\perp(1)}$ Collins FFs for the SIDIS + lattice fit (red and blue bands) at $Q^{2}=2 \mathrm{GeV}^{2}$, compared with the SIDIS-only fit uncertainties (yellow bands). The range of direct experimental constraints is indicated by the horizontal dashed lines. 


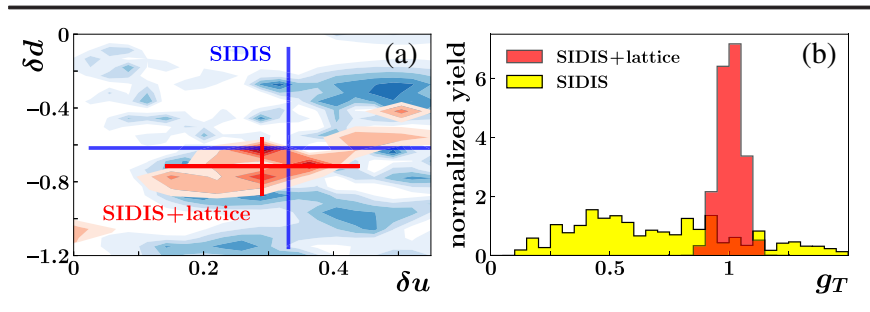

FIG. 3. (a) A contour plot of $\delta u$ and $\delta d$ samples from the MC analysis, for the SIDIS only (blue) and SIDIS + lattice (red) analysis. The expectation values and $1 \sigma$ uncertainties for both fits are indicated by the respective error bars. (b) Normalized yields for the isovector tensor charge $g_{T}$, for the SIDIS-only (yellow histograms) and SIDIS + lattice (red histograms) MC analyses.

unpolarized favored and unfavored FF widths. These values are compatible with ones found in the analysis by Anselmino et al. [54] of HERMES and COMPASS charged hadron multiplicities. On the other hand, the similar values found for the sea and valence PDF widths disagree with the chiral soliton model [55], for which the sea to valence ratio is $\sim 5$. Note also that while there appear some incompatibilities between the $x$ dependence of the HERMES and COMPASS $P_{h \perp}$-integrated $\pi^{ \pm}$multiplicities, our analysis uses only $P_{h \perp}$-dependent HERMES data that are given in bins of $x, z, Q^{2}$, and $P_{h \perp}$.

The transverse momentum widths for the valence and sea transversity PDFs are $\left\langle k_{\perp}^{2}\right\rangle_{h_{1}}^{q}=0.5(2) \mathrm{GeV}^{2}$ and $1.0(5) \mathrm{GeV}^{2}$, respectively, and $\left\langle p_{\perp}^{2}\right\rangle_{H_{1}^{\perp}}^{\pi / q}=0.12(4) \mathrm{GeV}^{2}$ and $0.06(3) \mathrm{GeV}^{2}$ for the favored and unfavored Collins FF widths, respectively. The relatively larger uncertainties on the $h_{1}$ and $H_{1}^{\perp}$ widths, compared with the unpolarized widths, reflect the higher precision of the HERMES multiplicity data, and the order of magnitude smaller number of data points for the Collins asymmetries.

Integrating the transversity PDFs over $x$, the resulting normalized yields from our MC analysis for the $\delta u$ and $\delta d$ moments are shown in Fig. 3, together with the isovector combination $g_{T}$. The most striking feature is the significantly narrower distributions evident when the SIDIS data are supplemented by the lattice $g_{T}$ input. The $u$ and $d$ tensor charges in Fig. 3(a), for example, change from $\delta u=$ $0.3(3) \rightarrow 0.3(2)$ and $\delta d=-0.6(5) \rightarrow-0.7(2)$ at the scale $Q^{2}=2 \mathrm{GeV}^{2}$, while the reduction in the uncertainty is even more dramatic for the isovector charge in Fig. 3(b), $g_{T}=0.9(8) \rightarrow 1.0(1)$. The earlier single-fit analysis of SIDIS data by Kang et al. [21] quotes $\delta u=0.39(11)$ and $\delta d=-0.22(14)$, with $g_{T}=0.61(25)$ at $Q^{2}=10 \mathrm{GeV}^{2}$, in apparent tension with the lattice results. This can be understood from Fig. 3(b), which demonstrates that the peak of the SIDIS-only distribution at $g_{T} \sim 0.5$ is consistent with the lower values found in earlier maximum likelihood analyses [10,21], but does not give a good representation of the mean value because of the long tail of the $g_{T}$ distribution.
Future extensions of this work will explore incorporating TMD evolution via the CSS framework [22,56], and the improved treatment of the large- $P_{h \perp}$ contributions through the addition of the $Y$ term [50]. The inclusion of $K^{ \pm}$SIDIS and $e^{+} e^{-}$annihilation data will allow further separation of sea quark flavor contributions to $h_{1}$ and better constraints on the favored and unfavored Collins FFs. Upcoming highprecision data from Jefferson Lab should also provide significantly improved kinematical coverage at intermediate $x$ and $z$ values.

We are grateful to J. Qiu for helpful comments. This work was supported by the U. S. Department of Energy Contract No. DE-AC05-06OR23177, under which Jefferson Science Associates, LLC operates Jefferson Lab, and by the National Science Foundation Contracts No. PHY-1623454, PHY-1653405, and PHY-1659177.

[1] J. Gao, L. Harland-Lang, and J. Rojo, arXiv:1709.04922.

[2] J. Blümlein, Prog. Part. Nucl. Phys. 69, 28 (2013).

[3] P. Jimenez-Delgado, W. Melnitchouk, and J. F. Owens, J. Phys. G 40, 093102 (2013).

[4] C. A. Aidala, S. D. Bass, D. Hasch, and G. K. Mallot, Rev. Mod. Phys. 85, 655 (2013).

[5] J. P. Ralston and D. E. Soper, Nucl. Phys. B152, 109 (1979).

[6] R. L. Jaffe and X.-D. Ji, Phys. Rev. Lett. 67, 552 (1991).

[7] J. L. Cortes, B. Pire, and J. P. Ralston, Z. Phys. C 55, 409 (1992).

[8] V. Barone, A. Drago, and P. G. Ratcliffe, Phys. Rep. 359, 1 (2002).

[9] A. Accardi et al., Eur. Phys. J. A 52, 268 (2016).

[10] Z. Ye, N. Sato, K. Allada, T. Liu, J.-P. Chen, H. Gao, Z.-B. Kang, A. Prokudin, P. Sun, and F. Yuan, Phys. Lett. B 767, 91 (2017).

[11] A. Accardi and A. Bacchetta, Phys. Lett. B 773, 632 (2017).

[12] M. Cirelli, E. D. Nobile, and P. Panci, J. Cosmol. Astropart. Phys. 10 (2013) 019.

[13] T. Bhattacharya, V. Cirigliano, S. D. Cohen, A. Filipuzzi, M. Gonzalez-Alonso, M. L. Graesser, R. Gupta, and H.-W. Lin, Phys. Rev. D 85, 054512 (2012).

[14] A. Courtoy, S. Baeßler, M. Gonzalez-Alonso, and S. Liuti, Phys. Rev. Lett. 115, 162001 (2015).

[15] J. C. Collins, Nucl. Phys. B396, 161 (1993).

[16] D. Boer, R. Jakob, and P. J. Mulders, Nucl. Phys. B504, 345 (1997).

[17] M. Anselmino, M. Boglione, U. D’Alesio, A. Kotzinian, F. Murgia, A. Prokudin, and C. Turk, Phys. Rev. D 75, 054032 (2007).

[18] M. Anselmino, M. Boglione, U. D’Alesio, A. Kotzinian, F. Murgia, A. Prokudin, and S. Melis, Nucl. Phys. B, Proc. Suppl. 191, 98 (2009).

[19] M. Anselmino, M. Boglione, U. D’Alesio, S. Melis, F. Murgia, and A. Prokudin, Phys. Rev. D 87, 094019 (2013).

[20] Z.-B. Kang, A. Prokudin, P. Sun, and F. Yuan, Phys. Rev. D 91, 071501 (2015).

[21] Z.-B. Kang, A. Prokudin, P. Sun, and F. Yuan, Phys. Rev. D 93, 014009 (2016). 
[22] J. Collins, Foundations of Perturbative QCD (Cambridge University Press, Cambridge, 2013).

[23] A. Bacchetta, A. Courtoy, and M. Radici, J. High Energy Phys. 03 (2013) 119.

[24] A. Bacchetta, A. Courtoy, and M. Radici, Phys. Rev. Lett. 107, 012001 (2011).

[25] M. Radici, A. Courtoy, A. Bacchetta, and M. Guagnelli, J. High Energy Phys. 05 (2015) 123.

[26] X. Ji, Phys. Rev. Lett. 110, 262002 (2013).

[27] J.-W. Chen, S. D. Cohen, X. Ji, H.-W. Lin, and J.-H. Zhang, Nucl. Phys. B911, 246 (2016).

[28] T. Bhattacharya, V. Cirigliano, S. D. Cohen, R. Gupta, H.-W. Lin, and B. Yoon, Phys. Rev. D 94, 054508 (2016).

[29] T. Bhattacharya, V. Cirigliano, S. D. Cohen, R. Gupta, A. Joseph, H.-W. Lin, and B. Yoon, Phys. Rev. D 92, 094511 (2015).

[30] J. R. Green, J. W. Negele, A. V. Pochinsky, S. N. Syritsyn, M. Engelhardt, and S. Krieg, Phys. Rev. D 86, 114509 (2012).

[31] Y. Aoki, T. Blum, H.-W. Lin, S. Ohta, S. Sasaki, R. Tweedie, J. Zanotti, and T. Yamazaki, Phys. Rev. D 82, 014501 (2010).

[32] A. Abdel-Rehim, C. Alexandrou, M. Constantinou, P. Dimopoulos, R. Frezzotti, K. Hadjiyiannakou, K. Jansen, C. Kallidonis, B. Kostrzewa, G. Koutsou, M. ManginBrinet, M. Oehm, G. C. Rossi, C. Urbach, and U. Wenger, Phys. Rev. D 92, 114513 (2015); 93, 039904(E) (2016).

[33] G. S. Bali, S. Collins, B. Glassle, M. Gockeler, J. Najjar, R. H. Rodl, A. Schafer, R. W. Schiel, W. Soldner, and A. Sternbeck, Phys. Rev. D 91, 054501 (2015).

[34] T. Yamazaki, Y. Aoki, T. Blum, H. W. Lin, M. F. Lin, S. Ohta, S. Sasaki, R. J. Tweedie, and J. M. Zanotti, Phys. Rev. Lett. 100, 171602 (2008).

[35] J. Skilling, Nested sampling for general Bayesian computation, http://www.inference.phy.cam.ac.uk/bayesys.

[36] P. Mukherjee, D. Parkinson, and A. R. Liddle, Astrophys. J. 638, L51 (2006).
[37] R. Shaw, M. Bridges, and M. P. Hobson, Mon. Not. R. Astron. Soc. 378, 1365 (2007).

[38] N. Sato, W. Melnitchouk, S. E. Kuhn, J. J. Ethier, and A. Accardi, Phys. Rev. D 93, 074005 (2016).

[39] J. J. Ethier, N. Sato, and W. Melnitchouk, Phys. Rev. Lett. 119, 132001 (2017).

[40] N. Sato, J. J. Ethier, W. Melnitchouk, M. Hirai, S. Kumano, and A. Accardi, Phys. Rev. D 94, 114004 (2016).

[41] J.-W. Chen and X. Ji, Phys. Lett. B 523, 107 (2001).

[42] W. Detmold, W. Melnitchouk, and A. W. Thomas, Phys. Rev. D 66, 054501 (2002).

[43] S. Aoki et al., Eur. Phys. J. C 77, 112 (2017).

[44] A. Bacchetta, M. Diehl, K. Goeke, A. Metz, P. J. Mulders, and M. Schlegel, J. High Energy Phys. 02 (2007) 093.

[45] J. C. Collins and D. E. Soper, Nucl. Phys. B193, 381 (1981); B213, 545(E) (1983).

[46] M. Anselmino, M. Boglione, U. D’Alesio, J. O. Gonzalez Hernandez, S. Melis, F. Murgia, and A. Prokudin, Phys. Rev. D 92, 114023 (2015).

[47] A. Airapetian et al., Phys. Lett. B 693, 11 (2010).

[48] M. Alekseev et al., Phys. Lett. B 673, 127 (2009).

[49] C. Adolph et al., Phys. Lett. B 744, 250 (2015).

[50] J. Collins, L. Gamberg, A. Prokudin, T. C. Rogers, N. Sato, and B. Wang, Phys. Rev. D 94, 034014 (2016).

[51] A. Accardi, L. T. Brady, W. Melnitchouk, J. F. Owens, and N. Sato, Phys. Rev. D 93, 114017 (2016).

[52] D. de Florian, R. Sassot, and M. Stratmann, Phys. Rev. D 76, 074033 (2007).

[53] A. Airapetian et al., Phys. Rev. D 87, 074029 (2013).

[54] M. Anselmino, M. Boglione, J. O. Gonzalez Hernandez, S. Melis, and A. Prokudin, J. High Energy Phys. 04 (2014) 005.

[55] P. Schweitzer, M. Strikman, and C. Weiss, J. High Energy Phys. 01 (2013) 163.

[56] J. C. Collins, D. E. Soper, and G. F. Sterman, Nucl. Phys. B250, 199 (1985). 